\title{
Hook-up of GluA2, GRIP and liprin- $\alpha$ for cholinergic muscarinic receptor-dependent LTD in the hippocampus Long-Jun $\mathrm{Wu}^{1}$, Yu-Tian Wang ${ }^{2}$ and Min Zhuo*1,3
}

\author{
Address: ${ }^{1}$ Department of Physiology, Faculty of Medicine, University of Toronto, 1 King's College Circle, Toronto, Ontario M5S 1A8, Canada, \\ ${ }^{2}$ Brain Research Centre, University of British Columbia, Vancouver, V6T 1Z3, Canada and ${ }^{3}$ Department of Brain and Cognitive Sciences, Seoul \\ National University, Seoul 151-746, Korea \\ Email: Long-Jun Wu - longjun.wu@utoronto.ca; Yu-Tian Wang - ytwang@interchange.ubc.ca; Min Zhuo* - min.zhuo@utoronto.ca \\ * Corresponding author
}

Published: 17 June 2009

Molecular Brain 2009, 2:17 doi:10.1186/1756-6606-2-17
Received: 26 May 2009

Accepted: 17 June 2009

This article is available from: http://www.molecularbrain.com/content/2/1/17

(c) 2009 Wu et al; licensee BioMed Central Ltd.

This is an Open Access article distributed under the terms of the Creative Commons Attribution License (http://creativecommons.org/licenses/by/2.0), which permits unrestricted use, distribution, and reproduction in any medium, provided the original work is properly cited.

\begin{abstract}
The molecular mechanism underlying muscarinic acetylcholine receptor-dependent LTD (mAChRLTD) in the hippocampus is less studied. In a recent study, a novel mechanism is described. The induction of $m A C h R-L T D$ required the activation of protein tyrosine phosphatase (PTP), and the expression was mediated by AMPA receptor endocytosis via interactions between GluA2, GRIP and liprin- $\alpha$. The hook-up of these proteins may result in the recruitment of leukocyte common antigen-related receptor (LAR), a PTP that is known to be involved in AMPA receptor trafficking. Interestingly, the similar molecular interaction cannot be applied to mGluR-LTD, despite the fact that the same G-protein involved in LTD is activated by both mAChR and mGluR. This discovery provides key molecular insights for cholinergic dependent cognitive function, and mAChR-LTD can serve as a useful cellular model for studying the roles of cholinergic mechanism in learning and memory.
\end{abstract}

\section{Editorial}

Activity-dependent changes in synaptic strength, such as long-term potentiation (LTP) and long-term depression (LTD), are thought to be the cellular models of learning and memory [1]. In the hippocampus, several mechanistically distinct forms of LTD have been reported. Two main forms of LTD (NMDA receptor (NMDAR)- and metabotropic glutamate receptor (mGluR)-dependent LTD), have been intensively studied at hippocampal CA1 region and the signaling pathways underlying LTD have been established. For example, NMDAR-LTD can be induced by prolonged low frequency stimulation (LFS). The mechanisms underlying NMDAR-LTD include activation of NMDA receptor, postsynaptic $\mathrm{Ca}^{2+}$ elevation, and subsequent activation of protein phosphatases. The AMPA receptor endocytosis is likely important for the expression of the depression. mGluR-LTD can be induced following paired-pulse LFS or DHPG (Group
I mGluR agonist) application, and is dependent on the activation of group I mGluR. Similar postsynaptic AMPA receptor endocytosis and possible presynaptic reduction of glutamate release are thought to contribute to reduced responses [2-6].

Less attention has been paid to another form of LTD, one which is dependent on muscarinic acetylcholine receptors (mAChR). First described in the visual cortex, mAChR-LTD was subsequently found in various brain regions, including the perirhinal cortex and hippocampus [7-11]. Cholinergic neurotransmission has been long implicated in memory and cognition [12] and thus mAChR-LTD is proposed to be critical for cholinergic-related brain functions and disease. However, the molecular mechanisms underlying mAChR-LTD remain largely unknown. Since mAChRs and mGluRs activate similar signaling pathways involving the same G-pro- 
teins and isoforms of phospholipase C (PLC) [13], it is reasonable to expect that mAChR-LTD and mGluR-LTD share similar molecular mechanisms. However, a recent study by Dickinson and colleagues [this issue, Dickinson et al., Molecular Brain] sheds new light on the molecular mechanisms underlying mAChR-LTD in the hippocampus. Using whole-cell patch clamp recording in hippocampal CA1 pyramidal neurons from 4-5 week-old young rats, they found that $\mathrm{mAChR}-\mathrm{LTD}$ requires the activation of $\mathrm{M} 1$ receptors and protein tyrosine phosphatases (PTPs), which in turn result in AMPA receptor internalization via interactions between GluA2, GRIP and liprin- $\alpha$. However, these same molecular interactions are not required for mGluR-LTD in the hippocampus.

The major cholinergic innervations of the hippocampus come from the medial septum [14]. Five different muscarinic receptor subtypes (M1-5) have been identified, all of them are expressed in the hippocampus. Among them, $\mathrm{M} 1,3,5$ are coupled to the Gq/11 and phospholipase C (PLC) pathway, while $\mathrm{M} 2$ and 4 receptors negatively regulate adenylyl cyclases [15]. In the hippocampal CA1 region, mAChR-LTD has only recently been identified. It is induced following application of the cholinergic muscarinic receptor agonist, carbachol (CCh) to hippocampal slices $[7,11]$. Here Cho and colleagues demonstrate that mAChR-LTD does not require the involvement of NMDA receptors. Moreover, mAChR-LTD occurs independently of mGluRs. These findings support the argument that mAchR-LTD represents a unique form of LTD within the hippocampus. Consistent with previous studies, M1 receptors were found to contribute to the induction of mAChR-LTD. This is demonstrated by the use of a selective M1 receptor agonist 77-LH-28-1, and the antagonist, pirenzepine. The cholinergic $M 1$ receptor is known to link to $\mathrm{Gq}$ and the subsequent PLC signaling pathway, which induces $\mathrm{Ca}^{2+}$ release from intracellular $\mathrm{Ca}^{2+}$ store and activation of PKC. To further test whether mAChR-LTD is $\mathrm{Ca}^{2+}{ }^{2+}$ or PKC-dependent, cyclopiazonic acid (for $\mathrm{Ca}^{2+}$ store depletion), BAPTA ( $\mathrm{Ca}^{2+}$ chelator), Ro 32-0432 (PKC inhibitor) and PKC19-31 (inhibitory peptide for PKC) were used and none was shown to affect mAChRLTD, providing the strong evidence that mAchR-LTD may employ special signaling pathways. Postsynaptic application of GDP $\beta$ S, a G-protein inhibitor, abolished mAChRLTD. Taken together, these results suggest that the induction of mAChR-LTD is dependent on M1 receptors and a G-protein signaling mechanism, but not on a conventional Gq-coupled pathway.

Protein phosphatases are known to contribute to hippocampal LTD [6]. For example, serine/threonine protein phosphatases PP1 and PP2B are required for NMDARLTD, while protein tyrosine phosphatase (PTP) is required for mGluR-LTD $[6,16,17]$. Therefore, Dickinson and colleagues set out to study whether these phosphatases play roles in mAChR-LTD. By postsynaptic application of phosphatase inhibitors, they found that PTP, but not serine/threonine protein phosphatases, is required for mAChR-LTD. In addition, they also found that protein synthesis is not required for mAChR-LTD. This result is quite surprising, since protein synthesis has been found to be required for both mGluR-LTD and mAChR-LTD in other studies $[7,10,18]$. It has been reported that extracellular signal-regulated kinase (ERK) and mammalian target of rapamycin (mTOR) translational activation pathways contribute to protein synthesis-dependent LTD $[7,19]$. Because different results have been reported in term of the requirement of protein synthesis in LTD, it would worthwhile in future studies to examine the roles of ERK and mTOR pathways in mAChR-LTD.

How then is mAChR-LTD expressed? AMPA receptor endocytosis is the key expression mechanism for both NMDAR-LTD and mGluR-LTD $[3,6,20,21]$. In a previous report, the surface GluA1 internalization has been observed following CCh treatment in cultured hippocampal neurons [7]. To examine the expression mechanism of mAChR-LTD, Dickinson et al. compared the cell surface and total expression level of GluA2 in control versus CChtreated hippocampal slices. Following $\mathrm{CCh}$ treatment they observed a reduction in GluA2 expression on the cell surface, while total expression levels of GluA2 remained largely unchanged. These results suggest a significant increase in GluA2-containing AMPA receptor endocytosis following LTD induction.

A number of studies have revealed that AMPA receptorinteracting proteins such as NSF, AP2, GRIP, ABP, and PICK1 are critically involved in AMPA receptor trafficking related synaptic plasticity [3,20,22]. Most notably, GRIP/ ABP and/or PICK1 are required for NMDAR-LTD in hippocampus, cerebellum and cortex, while PICK1 is required for mGluR-LTD in ventral tegmental area, cerebellum and perirhinal cortex $[3,22]$. However, it is still unknown which interacting proteins are involved in mAChR-LTD. To address this question, Dickinson et al. used peptide inhibitors to block either the interaction between GluA2 and PICK1 (pep2-EVKI), or between GluA2 and GRIP/ABP as well as PICK1 (pep2-SVKI). They found that pep2-SVKI, but not pep2-EVKI, inhibited mAChR-LTD, suggesting that GRIP rather than PICK1 is involved in mAChR-LTD. Interestingly, pep2-SVKI did not block mGluR-LTD, which suggests that neither GRIP nor PICK1 is involved. These findings indicate a different mechanism is likely involved at the level of AMPA receptor trafficking between mGluR- and mAChR-LTD.

To further examine the mechanisms by which GRIP modulates GluA2 trafficking and mAChR-LTD, they focused on the GRIP interacting protein, Liprin- $\alpha$, which can directly interact with GRIP via its PDZ6 domain [23]. They 
found that disrupting the interaction between GRIP and liprin- $\alpha$ using a synthetic peptide selectively blocked mAChR-LTD but not mGluR-LTD and NMDAR-LTD. These results suggest that the GRIP-liprin- $\alpha$ interaction is specifically required for mAChR-LTD. Liprin- $\alpha$ could recruit leukocyte common antigen-related receptors (LAR), a PTP known to be involved in AMPA receptor trafficking, axon guidance and neuronal development [24]. One attractive hypothesis is that activation of LAR phosphatase is triggered in mAChR-LTD via it's interaction with the liprin- $\alpha$-GRIP-GluA2 complex and the subsequent tyrosine dephosphorylation of GluA2. GluA2 tyrosine dephosphorylation results in the release of GluA2 from GRIP and AMPA receptor endocytosis, thereby expressing LTD. Indeed, in the present study, spectrum PTP inhibitors which can inhibit LAR phosphatase activity are effective in blocking mAChR-LTD. In addition, it has been reported that disruption of GRIP-liprin interactions, or knockdown of LAR interfere with dendritic AMPA receptor distribution $[23,24]$.

In summary, Dickinson et al. examined the detailed induction and expression mechanisms of mAChR-LTD in hippocampal CA1 region. They nicely demonstrated that cholinergic M1 receptors, G-protein signaling, PTP activity and GluA2 internalization are involved in mAChR-LTD. More importantly, their results reveal that the interaction of GluA2-GRIP-liprin- $\alpha$ is required for mAChR-LTD, the mechanisms of which may require the recruitment of LAR, GluA2 tyrosine dephosphorylation, and thus AMPA receptor endocytosis. The present study unveils a novel cellular mechanism for mAChR-LTD, which is different from mGluR-LTD. Future studies are needed to address how the activation of $\mathrm{M} 1$ receptor leads to the recruitment of LAR-liprin- $\alpha$-GRIP-GluA2 pathway. In addition, gene knockout mice deficient of AMPA receptor subunits can be used to evaluate/confirm the roles of these subunits in mAChR-LTD. Lastly, it would be valuable to identify whether the mechanisms observed in the present study are required for synaptically-induced mAChR-LTD in vivo and the pathophysiological relevance of heterosynaptic mAChR-LTD in brains under disease conditions.

\section{Acknowledgements}

Supported by grants from the EJLB-CIHR Michael Smith Chair in Neurosciences and Mental Health, Canada Research Chair, and World Class University (WCU) program to M. Z. L.-J.W. is supported by postdoctoral fellowships from the Canadian Institutes of Health Research and Fragile $\times$ Research Foundation of Canada.

\section{References}

I. Bliss TV, Collingridge GL: A synaptic model of memory: longterm potentiation in the hippocampus. Nature 1993, 36 I:31-39.

2. Toyoda H, Zhao MG, Zhuo M: NMDA receptor-dependent longterm depression in the anterior cingulate cortex. Rev Neurosci 2006, I 7:403-4I3.
3. Collingridge GL, Isaac JT, Wang YT: Receptor trafficking and synaptic plasticity. Nat Rev Neurosci 2004, 5:952-962.

4. Shepherd JD, Huganir RL: The cell biology of synaptic plasticity: AMPA receptor trafficking. Annu Rev Cell Dev Biol 2007, 23:613-643.

5. Bellone C, Luscher C, Mameli M: Mechanisms of synaptic depression triggered by metabotropic glutamate receptors. Cell $\mathrm{Mol}$ Life Sci 2008, 65:2913-2923.

6. Malenka RC, Bear MF: LTP and LTD: an embarrassment of riches. Neuron 2004, 44:5-21.

7. Volk LJ, Pfeiffer BE, Gibson JR, Huber KM: Multiple Gq-coupled receptors converge on a common protein synthesis-dependent long-term depression that is affected in fragile $x$ syndrome mental retardation. J Neurosci 2007, 27: I I624-II634.

8. Jo J, Ball SM, Seok H, Oh SB, Massey PV, Molnar E, Bashir ZI, Cho K: Experience-dependent modification of mechanisms of longterm depression. Nat Neurosci 2006, 9:170-172.

9. Kirkwood A, Rozas C, Kirkwood J, Perez F, Bear MF: Modulation of long-term synaptic depression in visual cortex by acetylcholine and norepinephrine. J Neurosci 1999, 19:1599-1609.

10. Massey PV, Bhabra G, Cho K, Brown MW, Bashir Zl: Activation of muscarinic receptors induces protein synthesis-dependent long-lasting depression in the perirhinal cortex. Eur J Neurosci 200I, I 4: I45-I52.

II. Scheiderer CL, McCutchen E, Thacker EE, Kolasa K, Ward MK, Parsons D, Harrell LE, Dobrunz LE, McMahon LL: Sympathetic sprouting drives hippocampal cholinergic reinnervation that prevents loss of a muscarinic receptor-dependent long-term depression at CA3-CAI synapses. J Neurosci 2006, 26:3745-3756.

12. Sarter M, Parikh V: Choline transporters, cholinergic transmission and cognition. Nat Rev Neurosci 2005, 6:48-56.

13. Bashir ZI: On long-term depression induced by activation of G-protein coupled receptors. Neurosci Res 2003, 45:363-367.

14. Bolam JP, Hanley JJ, Booth PA, Bevan MD: Synaptic organisation of the basal ganglia. J Anat 2000, 196(Pt 4):527-542.

15. Venter JC, Fraser CM, Kerlavage AR, Buck MA: Molecular biology of adrenergic and muscarinic cholinergic receptors. A perspective. Biochem Pharmacol 1989, 38:1 197-1208.

16. Kameyama K, Lee HK, Bear MF, Huganir RL: Involvement of a postsynaptic protein kinase $A$ substrate in the expression of homosynaptic long-term depression. Neuron 1998, 21:1163-II75.

17. Moult PR, Gladding CM, Sanderson TM, Fitzjohn SM, Bashir ZI, Molnar E, Collingridge GL: Tyrosine phosphatases regulate AMPA receptor trafficking during metabotropic glutamate receptor-mediated long-term depression. J Neurosci 2006, 26:2544-2554.

18. Waung MW, Pfeiffer BE, Nosyreva ED, Ronesi JA, Huber KM: Rapid translation of Arc/Arg3.I selectively mediates mGluRdependent LTD through persistent increases in AMPAR endocytosis rate. Neuron 2008, 59:84-97.

19. Scheiderer CL, Smith CC, McCutchen E, McCoy PA, Thacker EE, Kolasa K, Dobrunz LE, McMahon LL: Coactivation of M(I) muscarinic and alpha $I$ adrenergic receptors stimulates extracellular signal-regulated protein kinase and induces long-term depression at CA3-CAI synapses in rat hippocampus. J Neurosci 2008, 28:5350-5358.

20. Malinow R, Malenka RC: AMPA receptor trafficking and synaptic plasticity. Annu Rev Neurosci 2002, 25: I03-126.

21. Carroll RC, Beattie EC, von Zastrow M, Malenka RC: Role of AMPA receptor endocytosis in synaptic plasticity. Nat Rev Neurosci 200I, 2:3I5-324.

22. Isaac JT, Ashby M, McBain C]: The role of the GluR2 subunit in AMPA receptor function and synaptic plasticity. Neuron 2007, 54:859-87I.

23. Wyszynski M, Kim E, Dunah AW, Passafaro M, Valtschanoff JG, SerraPages C, Streuli M, Weinberg RJ, Sheng M: Interaction between GRIP and liprin-alpha/SYD2 is required for AMPA receptor targeting. Neuron 2002, 34:39-52.

24. Dunah AW, Hueske E, Wyszynski M, Hoogenraad CC, Jaworski J, Pak DT, Simonetta A, Liu G, Sheng M: LAR receptor protein tyrosine phosphatases in the development and maintenance of excitatory synapses. Nat Neurosci 2005, 8:458-467. 